\title{
Analysis of Directional Logging Tools in Anisotropic and Multieccentric Cylindrically-Layered Earth Formations
}

\author{
Guo-Sheng Liu, Fernando L. Teixeira, and Guo-Ji Zhang
}

\begin{abstract}
We develop a pseudoanalytical method for the analysis of directional resistivity well-logging tools consisting of multiple tilted-coil antennas in cylindrically-layered Earth formations which have anisotropic conductivities and where each layer may be eccentric to the others (multieccentric formations). The cylindrically-layered, anisotropic, and multieccentric scenarios considered here are often encountered in deviated/horizontal drilling for hydrocarbon exploration, where the logging tool may be eccentric to the borehole due to mechanical vibrations and/or gravitational pull, and where eccentric invaded zones (of mud filtrate into the formation) may also arise because of gravitational pull on the mud filtrate.
\end{abstract}

Index Terms-Anisotropic media, geophysical exploration, logging-while-drilling (LWD).

\section{INTRODUCTION}

D IRECTIONAL well-logging resistivity tools are routinely used in hydrocarbon exploration to determine, for example, horizontal and vertical resistivities in anisotropic Earth formations and relative dip angle during deviated drilling [1]-[5]. For logging-while-drilling (LWD) tools, tiltedcoil antennas provide directional data for real-time geosteering. Resistivity tools typically consist of multiple tilted-coil antennas wrapped around a cylindrical metallic mandrel. A transmitter coil excites eddy currents in the surrouding Earth formation [6]. This current is proportional to the formation conductivity and produces a secondary electric field and induced voltage in the receivers. Various techniques have been proposed to model this problem. These include brute-force numerical methods such as finite-differences (FD) [7]-[10] and finite elements (FE) [11]-[13], and pseudoanalytical approaches [4], [5], [14]-[19]. The former can handle arbitrarily complex Earth formations but suffer from high computational costs. The latter are less flexible but less computationally costly. Many pseudoanalytical approaches approximate coil antennas as magnetic dipoles, which ignores mandrel effects and finite-size antenna effects. Pseudoanalytical approaches

Manuscript received MM/DD/YYYY; revised MM/DD/YYYY. This work was supported in part by OSC under grants PAS-0061 and PAS-0110, and by the Chinese Scholarship Council under grant 2008615058.

G.-S. Liu is with the Department of Information Management Engineering, Guangdong University of Technology, Guangzhou 510520, China (email: armstrongliu@yahoo.cn).

F. L. Teixeira is with the ElectroScience Laboratory and Department of Electrical and Computer Engineering, The Ohio State University, Columbus, OH 43212 USA (email: teixeira@ece.osu.edu).

G.-J. Zhang is with the Department of Mathematics, South China University of Technology (SCUT), Guangzhou 510641, China (email: magjzh@scut.edu.cn) that do include these two effects [4], [5] have attracted much interest in recent years as they provide better accuracy.

Lovell and Chew [14] studied the response of a centered dipole source in a cylindrically-layered formation, and later extended it to horizontal eccentric coil antenna sources [15]. Hagiwara et al. [4] extended this approach to arbitrarilytilted coils, and Hue et al. [5] to eccentric and arbitrarilytilted coils. All these references have assumed concentric isotropic cylindrical layers in the Earth formation, i.e., the only eccentricity, if any, is that of the logging tool itself.

In a number of practical scenarios, it is of interest to study "multieccentric" formations where each successive cylindrical layer may have a different axis of symmetry, as illustrated in Fig. 5. This geometry may arise due to (1) mechanical vibrations and/or gravitational pull causing the logging tool to become eccentric with respect to the borehole and (2) the presence of eccentric invasion zones of mud filtrate (borehole fluid) in the formation. The eccentricity of the latter may be a consequence of gravitational pull during deviated or horizontal drilling ${ }^{1}$. The analysis of multieccentric formations is also useful to model, as a limit case, the response of logging tools near lateral beds, see Fig. 8. Finally, consideration of anisotropy is important because Earth formations often have different conductivities along horizontal and vertical directions [6], [9]. Indeed, this consideration is one of the original motivations for employing tilted-coil antennas [2], [20], [21].

In this work, we extend the pseudoanalytical approach of [5] to model anisotropic and multieccentric Earth formations. The formalism employs cylindrical eigenfunctions [22], [23] in anisotropic media to expand the field in each cylindrical layer. Generalized reflection and transmission matrices for multiple cylindrical layers are derived in a recursive fashion from single-interface ones. The addition theorem [22] for cylindrical waves is applied in a nested fashion to model multieccentric layers.

\section{Cylindrical Eigenfunctions in Uniaxial Media}

In this Section, we write down the field solutions for Maxwell's equations in terms of cylindrical eigenfunctions by extending the basic approach outlined in [5] to uniaxial anisotropic media ${ }^{2}$. Assuming a time-harmonic dependence of

\footnotetext{
${ }^{1}$ In practice, filtrate invasions are expected to deviate from a circular shape, but an eccentric circular layer serves as a good first approximation of the slumping effect of gravity.

${ }^{2}$ Note that the notation adopted here differs from that in [5] in some places.
} 
the form $e^{-i \omega t}$, we write Maxwell's equations as [22]

$$
\begin{aligned}
\nabla \times \overrightarrow{\mathcal{E}} & =i \omega \mu \overrightarrow{\mathcal{H}} \\
\nabla \times \overrightarrow{\mathcal{H}} & =-i \omega \overline{\bar{\epsilon}} \cdot \overrightarrow{\mathcal{E}}+\overrightarrow{\mathcal{J}}
\end{aligned}
$$

where $\overrightarrow{\mathcal{E}}$ and $\overrightarrow{\mathcal{H}}$ are the electric and magnetic fields respectively, $\overrightarrow{\mathcal{J}}$ is the causative electric current density, $\mu$ is the permeability, and $\overline{\bar{\epsilon}}$ is the complex permittivity tensor. We consider a conductivity tensor expressed in cylindrical coordinates as $\overline{\bar{\epsilon}}=\epsilon+i \overline{\bar{\sigma}} / \omega$ with $\overline{\bar{\sigma}}=\operatorname{diag}\left\{\sigma_{\rho}, \sigma_{\phi}, \sigma_{z}\right\}$, and where $\sigma_{\rho}=\sigma_{\phi}$ is the conductivity in transverse plane (denoted by $\sigma_{s}$ ), so that $\overline{\bar{\epsilon}}=\operatorname{diag}\left\{\epsilon_{s}, \epsilon_{s}, \epsilon_{z}\right\}$. We next employ a Fourier transformation

$$
\overrightarrow{\mathcal{E}}(\rho, \phi, z)=\frac{1}{2 \pi} \int_{-\infty}^{+\infty} d k_{z} e^{i k_{z} z} \vec{E}\left(\rho, \phi, k_{z}\right),
$$

and decompose the problem into axial and transverse components as

$$
\vec{E}=\vec{E}_{s}+\hat{z} E_{z} \quad \nabla=\nabla_{s}+\hat{z} i k_{z},
$$

and similarly for the other fields, so that Maxwell's equations are rewritten as

$$
\begin{array}{r}
i k_{z} \hat{z} \times \vec{E}_{s}+\nabla_{s} \times \hat{z} E_{z}=i \omega \mu \vec{H}_{s} \\
i k_{z} \hat{z} \times \vec{H}_{s}+\nabla_{s} \times \hat{z} H_{z}=-i \omega \epsilon_{s} \vec{E}_{s}+\vec{J}_{s} \\
\nabla_{s} \times \vec{E}_{s}=i \omega \mu \hat{z} H_{z} \\
\nabla_{s} \times \vec{H}_{s}=-i \omega \epsilon_{z} \hat{z} E_{z}+\hat{z} J_{z} .
\end{array}
$$

Using $\hat{z} \times\left(\nabla_{s} \times \hat{z} F_{z}\right)=\nabla_{s} F_{z}$ and $\hat{z} \times\left(\hat{z} \times \vec{F}_{s}\right)=-\vec{F}_{s}$, and after a few manipulations, we have in a source-free region

$$
\vec{E}_{s}=\frac{1}{k_{\rho}^{2}}\left(i k_{z} \nabla_{s} E_{z}+i \omega \mu \nabla_{s} \times \hat{z} H_{z}\right)
$$

and a dual equation for $\vec{H}_{s}$, where $k_{\rho}=\sqrt{k^{2}-k_{z}^{2}}, k^{2}=$ $\omega^{2} \mu \epsilon_{s}$, and $\Im m\left[k_{\rho}\right]>0$ [24]. Substituting (4) into (3) and using the expansion

$$
E_{z}\left(\rho, \phi, k_{z}\right)=\sum_{\nu=-\infty}^{+\infty} e^{i \nu \phi} e_{z \nu}\left(\rho, k_{z}\right),
$$

and similarly for $H_{z}$, we obtain the following ordinary differential equations

$$
\begin{aligned}
\frac{1}{\rho} \frac{d}{d \rho}\left(\rho \frac{d e_{z \nu}}{d \rho}\right)+\left(\frac{\epsilon_{z}}{\epsilon_{s}} k_{\rho}^{2}-\frac{\nu^{2}}{\rho^{2}}\right) e_{z \nu} & =0 \\
\frac{1}{\rho} \frac{d}{d \rho}\left(\rho \frac{d h_{z \nu}}{d \rho}\right)+\left(k_{\rho}^{2}-\frac{\nu^{2}}{\rho^{2}}\right) h_{z \nu} & =0 .
\end{aligned}
$$

Eqs. (6) can be recognized as different versions of Bessel equation, which admit general solutions of the form

$$
\left[\begin{array}{c}
e_{z \nu} \\
h_{z \nu}
\end{array}\right]=\overline{\bar{H}}_{z \nu}^{(1)}\left(k_{\rho} \rho, \alpha\right) \bar{a}_{\nu}+\overline{\bar{J}}_{z \nu}\left(k_{\rho} \rho, \alpha\right) \bar{b}_{\nu}
$$

where

$$
\overline{\bar{G}}_{z \nu}\left(k_{\rho} \rho, \alpha\right)=\left[\begin{array}{cc}
G_{\nu}\left(\alpha k_{\rho} \rho\right) & 0 \\
0 & G_{\nu}\left(k_{\rho} \rho\right)
\end{array}\right], \quad \alpha=\sqrt{\epsilon_{z} / \epsilon_{s}}
$$

with $G_{\nu}=J_{\nu}$ or $H_{\nu}^{(1)}$ being the Bessel function of first kind and the Hankel function of first kind, respectively. From physical considerations, the square root for $\alpha$ is chosen such

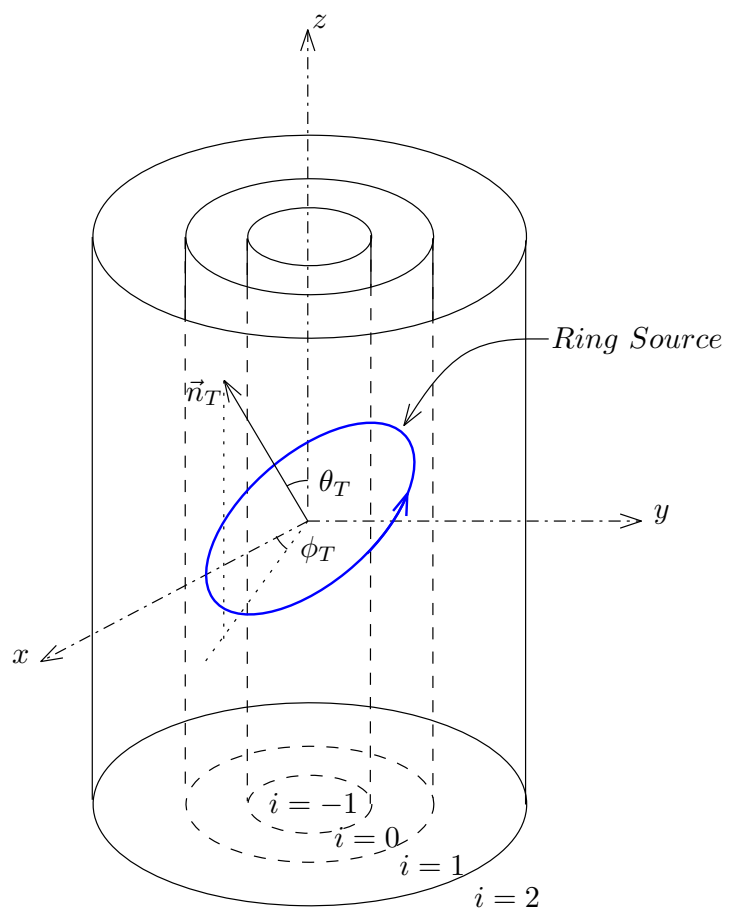

Fig. 1. Geometry of a tilted-coil antenna in a cylindrically multilayered formation, with tilt angle $\left(\theta_{T}, \phi_{T}\right)$. The coil antenna is located within the $i=0$ layer (borehole). The first layer $i=-1$ represents to the metallic mandrel. The vector $\vec{n}_{T}$ represents the normal unit vector to the plane of the coil antenna. Additional layers $i=1,2, \ldots$ can be included to model complex Earth formations including invaded zones.

that $\Im m\left[\alpha k_{\rho}\right]>0$ [24]. The amplitude coefficients $\bar{a}_{\nu}$ and $\bar{b}_{\nu}$ are generic $2 \times 1$ column vectors to be determined from the boundary conditions. Since only Hankel functions of the first kind are used in what follows, we will drop the superscript in $H_{\nu}^{(1)}$ for notational simplicity.

The transverse field components can be obtained by substituting (7) into (4). In the case of logging tools with coil antennas, the $\rho$-component of the field does not contribute to the voltage at receiver coil since the tangent vector to the coil(s) does not have a radial component. The $\phi$-components can be written as

$$
\left[\begin{array}{l}
e_{\phi \nu} \\
h_{\phi \nu}
\end{array}\right]=\overline{\bar{H}}_{\phi \nu}\left(k_{\rho} \rho, \alpha\right) \bar{a}_{\nu}+\overline{\bar{J}}_{\phi \nu}\left(k_{\rho} \rho, \alpha\right) \bar{b}_{\nu}
$$

where

$\overline{\bar{G}}_{\phi \nu}\left(k_{\rho} \rho, \alpha\right)=\frac{1}{k_{\rho}^{2} \rho}\left[\begin{array}{cc}-\nu k_{z} G_{\nu}\left(\alpha k_{\rho} \rho\right) & -i \omega \mu k_{\rho} \rho G_{\nu}^{\prime}\left(k_{\rho} \rho\right) \\ i \omega \epsilon_{s} \alpha k_{\rho} \rho G_{\nu}^{\prime}\left(\alpha k_{\rho} \rho\right) & -\nu k_{z} G_{\nu}\left(k_{\rho} \rho\right)\end{array}\right]$.

where the prime denotes derivative with respect to the argument.

\section{TILTED-COIL SOURCES IN ANISOTROPIC, MultiecCentric LAyered Media}

Fig. 1 depicts a tilted coil in a multilayered cylindrical media. In the case of directional logging-tools, this geometry includes a metallic mandrel $(i=-1)$, the borehole $(i=0)$, and the Earth formation $(i=1,2,3 \ldots$ layers). To conform to the practical scenarios of interest, we assume the first two layers isotropic, and the remaining possibly anisotropic. 
The layer $i=1$ may refer to an invaded zone of mud filtrate (borehole fluid) into the adjacent Earth formation. The boundary between the $i$ th layer and $(i+1)$ th layer is denoted by $R_{i}$. The coordinates of the transmitter coil antenna are given by $\vec{r}_{T}=\hat{\rho} \rho_{T}-\hat{z}\left(z_{T}+\rho_{T} \tan \theta_{T} \cos \left(\phi-\phi_{T}\right)\right)$, where $\rho_{T}$ is the radial coordinate of the coil, $z_{T}$ is the vertical coordinate of the center of the coil, $\theta_{T}$ is the elevation tilt angle, and $\phi_{T}$ is the azimuthal direction of the tilt [5]. The coordinates of receiver antennas are defined similarly. A prototypical directional logging tool would have one transmitter coil antenna and two receiver coil antennas. The current density on the transmitter antenna is given by ${ }^{3}$

$$
\overrightarrow{\mathcal{J}}=I_{0} \delta\left(\rho-r_{T}\right) \delta\left(z-z_{T}-\sin \left(\phi-\phi_{T}\right) \tan \theta_{T}\right) \vec{a}_{T}
$$

where $I_{0}$ is the current source amplitude and $\vec{a}_{T}$ is the tangent vector to the tilted-coil antenna plane given by $\vec{a}_{T}=\vec{a}_{\phi}+$ $\sin \left(\phi-\phi_{T}\right) \tan \theta_{T} \vec{a}_{z}$.

During drilling, the transmitter coil is excited by an sinusoidal waveform, and phase difference and amplitude ratio between the voltages excited at the receiver coils are measured. The voltage at a receiver coil for a unit current source at the transmitter (transimpedance) can be written as [5]

$$
V=\int_{-\infty}^{+\infty} d k_{z} e^{i k_{z}\left(z_{R}-z_{T}\right)} \omega\left(k_{z}\right),
$$

where

$$
\begin{aligned}
\omega\left(k_{z}\right)=-\rho_{R} \sum_{\nu=-\infty}^{+\infty} e^{i \nu\left(\phi_{R}-\phi_{T}\right)} & \times\left[d_{T E} e_{\phi \nu}\left(\rho_{R}, k_{z}\right)+d_{T M} e_{z \nu}\left(\rho_{R}, k_{z}\right)\right],
\end{aligned}
$$

and $d_{T E}=i^{-\nu} J_{\nu}\left(\kappa_{R}\right), d_{T M}=-\nu i^{-\nu} J_{\nu}\left(\kappa_{R}\right) /\left(k_{z} \rho_{R}\right)$, and $\kappa_{R}=k_{z} \rho_{R} \tan \left(\theta_{R}\right)$. Details on the derivation of the above expression are found in [5], [25] $]^{4}$. The analysis in [5] was limited to isotropic media and one single eccentric layer. In the following, we lift these two restrictions.

We can express the solutions in the borehole layer $i=0$, for computing the transimpedance in (10), as

$$
\left[\begin{array}{c}
e_{z \nu} \\
h_{z \nu}
\end{array}\right]= \begin{cases}{\left[\overline{\bar{H}}_{z \nu}\left(k_{\rho} \rho, \alpha\right) \overline{\bar{M}}_{\nu 0}+\overline{\bar{J}}_{z \nu}\left(k_{\rho} \rho, \alpha\right)\right] \bar{b}_{\nu 0}} & \rho \leq \rho_{T} \\
{\left[\overline{\bar{H}}_{z \nu}\left(k_{\rho} \rho, \alpha\right)+\overline{\bar{J}}_{z \nu}\left(k_{\rho} \rho, \alpha\right) \overline{\bar{N}}_{\nu 0}\right] \bar{a}_{\nu 0}} & \rho>\rho_{T}\end{cases}
$$

with similar expressions for $e_{\phi \nu}$ and $h_{\phi \nu}$, where $\overline{\bar{M}}_{\nu 0}$ and $\overline{\bar{N}}_{\nu 0}$ are generalized reflection and transmission matrices ${ }^{5}$, as derived in Appendix A, and the coefficients $\bar{a}_{\nu 0}$ and $\bar{b}_{\nu 0}$ can be obtained from the field produced by the (normalized) current source (9) in a homogeneous medium (incident field), as detailed in Appendix B (see also [5]). In multilayered media, $k_{\rho}$ and $\alpha$ in expressions such as (12) vary from layer to layer.

${ }^{3}$ Note that $\vec{a}_{T}$ is not a unit vector, and that $\nabla \cdot \overrightarrow{\mathcal{J}}=0$. Even though the magnitude of the current density $\overrightarrow{\mathcal{J}}$ varies with $\phi$, the flux of it over any (infinitesimal) cross section (and hence the total current flowing along the coil) is constant and equal to $I_{0}$.

${ }^{4}$ The integral in [5] is a folded (less general) version of the above.

${ }^{5}$ The terminology "reflection" and "transmission" is used in a loose sense here. More precisely, these coefficient matrices refer to the ratio between standing wave and outgoing wave amplitudes in each of the layers $i$, and vice-versa.

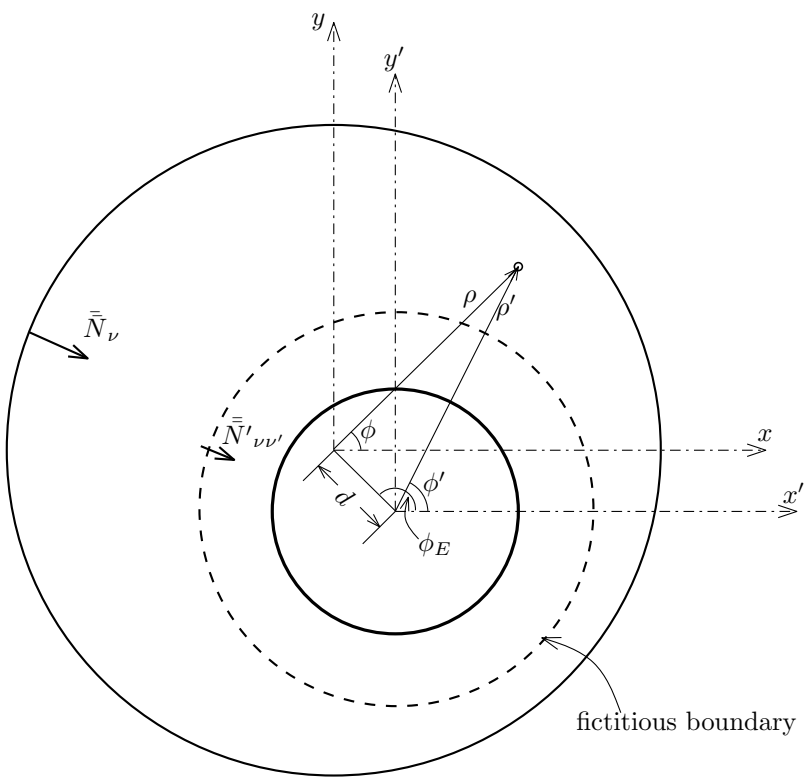

Fig. 2. Cross-section view of an eccentric borehole geometry in cylindrical coordinates. The nonprimed coordinate system $(\rho, \phi)$ is concentric to the outer circular boundary and has a displacement of $\left(d, \phi_{E}\right)$ with respect to the primed coordinate system $\left(\rho^{\prime}, \phi^{\prime}\right)$, which is concentric to the inner circular boundary. The fictitious circular boundary shown by a dashed circle is also concentric to the primed coordinate system.

To simplify the notation, we drop layer indices in $k_{\rho}$ and $\alpha$ except when necessary to distinguish them.

\section{A. Multieccentric Anisotropic Layers}

Consider two eccentric cylindrical layers as shown by the solid line circles in Fig. 2. The centers of the inner and outer layers coincide with the origins of primed $\left(\rho^{\prime}, \phi^{\prime}\right)$ and nonprimed $(\rho, \phi)$ coordinate systems, respectively. The nonprimed coordinates can be treated as a displacement of $\left(d, \phi_{E}\right)$ on the primed coordinate.

Consider the fields at a certain point between the two circles. Using (2) and (5), the spectral components $E_{z}$ and $H_{z}$ can be expressed as

$$
\left[\begin{array}{l}
E_{z} \\
H_{z}
\end{array}\right]=\sum_{\nu=-\infty}^{+\infty} e^{i \nu \phi^{\prime}}\left[\overline{\bar{H}}_{z \nu}\left(k_{\rho} \rho^{\prime}, \alpha\right) \bar{a}_{\nu}^{\prime}+\overline{\bar{J}}_{z \nu}\left(k_{\rho} \rho^{\prime}, \alpha\right) \bar{b}_{\nu}^{\prime}\right]
$$

in the primed coordinate, or

$$
\left[\begin{array}{l}
E_{z} \\
H_{z}
\end{array}\right]=\sum_{\nu=-\infty}^{+\infty} e^{i \nu \phi}\left[\overline{\bar{H}}_{z \nu}\left(k_{\rho} \rho, \alpha\right) \bar{a}_{\nu}+\overline{\bar{J}}_{z \nu}\left(k_{\rho} \rho, \alpha\right) \bar{b}_{\nu}\right]
$$

in the nonprimed coordinate. Applying Graf's addition theorem for cylindrical waves [22], we have

$$
\begin{array}{r}
\overline{\bar{G}}_{z \nu}\left(k_{\rho} \rho, \alpha\right) e^{i \nu \phi}=\sum_{\nu^{\prime}=-\infty}^{+\infty} \overline{\bar{G}}_{z \nu^{\prime}}\left(k_{\rho} \rho^{\prime}, \alpha\right) e^{i \nu^{\prime} \phi^{\prime}} \\
\times \overline{\bar{J}}_{z, \nu^{\prime}-\nu}\left(k_{\rho} d, \alpha\right) e^{-i\left(\nu^{\prime}-\nu\right) \phi_{E}}
\end{array},
$$

whereby the fields in nonprimed coordinate are expressed in terms of the eigenfunctions in the primed coordinate. This 
leads to the following transformation rules between field coefficients in the two coordinate systems

$$
\left\{\begin{array}{l}
\bar{a}_{\nu^{\prime}}^{\prime}=\sum_{\nu=-\infty}^{+\infty} \overline{\bar{J}}_{z, \nu^{\prime}-\nu}\left(k_{\rho} d, \alpha\right) e^{-i\left(\nu^{\prime}-\nu\right) \phi_{E}} \bar{a}_{\nu} \\
\bar{b}_{\nu^{\prime}}^{\prime}=\sum_{\nu=-\infty}^{+\infty} \overline{\bar{J}}_{z, \nu^{\prime}-\nu}\left(k_{\rho} d, \alpha\right) e^{-i\left(\nu^{\prime}-\nu\right) \phi_{E}} \bar{b}_{\nu}
\end{array}\right.
$$

We next introduce a fictitious boundary, represented by the dashed circle in Fig. 2 and concentric to the inner layer. Note that the fictitious boundary is chosen in a different way than in [5], [15]. This choice facilitates the treatment of multiple eccentric layers to be considered later in this Section and the derivation of an iterative solution for the field coefficients as detailed in Appendix B. Some further numerical considerations are discussed in Appendix C.

Different azimuthal modes do couple when an outgoing wave from the inner layer is "reflected" by the fictitious boundary (due to the effect of the outer eccentric boundary). The equivalent "reflection" matrix $\overline{\bar{N}}_{\nu \nu^{\prime}}^{\prime}$ relating $\bar{b}_{\nu}^{\prime}$ and $\bar{a}_{\nu}^{\prime}$ is defined by

$$
\bar{b}_{\nu}^{\prime}=\sum_{\nu^{\prime}=-\infty}^{+\infty} \overline{\bar{N}}_{\nu \nu^{\prime}}^{\prime} \bar{a}_{\nu^{\prime}}^{\prime}
$$

On the other hand, the reflection matrix $\overline{\bar{N}}_{\nu}$ at the outer(most) boundary can be written independently for each mode in the nonprimed representation, as before, through

$$
\bar{b}_{\nu}=\overline{\bar{N}}_{\nu} \bar{a}_{\nu}
$$

Substituting (17) and (18) into (16) leads to

$$
\begin{array}{r}
\sum_{\nu^{\prime}=-\infty}^{+\infty} \overline{\bar{N}}_{\nu \nu^{\prime}}^{\prime} \sum_{\nu^{\prime \prime}=-\infty}^{+\infty} \overline{\bar{J}}_{z, \nu^{\prime}-\nu^{\prime \prime}}\left(k_{\rho} d, \alpha\right) e^{-i\left(\nu^{\prime}-\nu^{\prime \prime}\right) \phi_{E}} \bar{a}_{\nu^{\prime \prime}} \\
=\sum_{\nu^{\prime}=-\infty}^{+\infty} \overline{\bar{J}}_{z, \nu-\nu^{\prime}}\left(k_{\rho} d, \alpha\right) e^{-i\left(\nu-\nu^{\prime}\right) \phi_{E}} \overline{\bar{N}}_{\nu^{\prime}} \bar{a}_{\nu^{\prime}}
\end{array}
$$

Eq. (19) can be solved for $\overline{\bar{N}}_{\nu \nu^{\prime}}^{\prime}$ since $\bar{a}_{\nu}$ is arbitrary. Since [26]

$$
\sum_{\nu^{\prime}=-\infty}^{+\infty} J_{\nu+\nu^{\prime}}(u) J_{\nu^{\prime}}(u)=J_{\nu}(0)=\delta_{\nu 0}
$$

where $\delta_{\nu 0}$ is the Kronecker delta, a convenient choice is $\bar{a}_{\nu^{\prime \prime}}=$ $\overline{\bar{J}}_{z, \nu^{\prime}-\nu^{\prime \prime}}\left(k_{\rho} d, \alpha\right) e^{-i\left(\nu^{\prime \prime}-\nu^{\prime}\right) \phi_{E}}$ so that

$$
\begin{aligned}
\overline{\bar{N}}_{\nu \nu^{\prime}}^{\prime}= & e^{-i\left(\nu-\nu^{\prime}\right) \phi_{E}} \\
& \times \sum_{\nu^{\prime \prime}=-\infty}^{+\infty} \overline{\bar{J}}_{z, \nu-\nu^{\prime \prime}}\left(k_{\rho} d, \alpha\right) \overline{\bar{N}}_{\nu^{\prime \prime}} \overline{\bar{J}}_{z, \nu^{\prime}-\nu^{\prime \prime}}\left(k_{\rho} d, \alpha\right) .
\end{aligned}
$$

In this fashion, a geometry of two eccentric layers has been transformed into a concentric geometry by introducing a fictitious boundary.

In the case of multiple eccentric layers, the procedure above is simply iterated, starting from the outermost layer and moving towards the inner layers. Suppose outer layer $i$ and inner layer $i-1$ are eccentric to each other, with nonprimed and primed coordinates centered on $i$ and $i-1$, respectively. In this case, the equivalent relations to (17) and (18) are

$$
\begin{aligned}
& \bar{b}_{\nu}^{\prime}=\sum_{\nu^{\prime}=-\infty}^{+\infty} \overline{\bar{N}}_{\nu \nu^{\prime}, i}^{\prime} \bar{a}_{\nu^{\prime}}^{\prime}, \\
& \bar{b}_{\nu}=\sum_{\nu^{\prime}=-\infty}^{+\infty} \overline{\bar{N}}_{\nu \nu^{\prime}, i} \bar{a}_{\nu^{\prime}},
\end{aligned}
$$

where $\overline{\bar{N}}_{\nu \nu^{\prime}, i}$ is the known "reflection" matrix from layer $i$, similarly to $\overline{\bar{N}}_{\nu}$ in (18). Note that, as opposed to (18), the matrix in (23) in general couples azimuthal modes because of the possible presence of eccentric layers external to layer $i$.

Following the addition theorem procedure delineated above, the reflection layer $\overline{\bar{N}}_{\nu \nu^{\prime}, i}^{\prime}$ from a fictitious boundary, within layer $i$, that is external and concentric to layer $i-1$ can now be obtained from $\overline{\bar{N}}_{\nu \nu^{\prime}, i}$ as

$$
\begin{aligned}
\overline{\bar{N}}_{\nu \nu^{\prime}, i}^{\prime}= & \sum_{\nu^{\prime \prime}=-\infty}^{+\infty} \overline{\bar{J}}_{z, \nu-\nu^{\prime \prime}}\left(k_{\rho} d, \alpha\right) e^{-i\left(\nu-\nu^{\prime \prime}\right) \phi_{E}} \\
& \times \sum_{\nu^{\prime \prime \prime}=-\infty}^{+\infty} \overline{\bar{N}}_{\nu^{\prime \prime} \nu^{\prime \prime \prime}, i} \overline{\bar{J}}_{z, \nu^{\prime}-\nu^{\prime \prime \prime}}\left(k_{\rho} d, \alpha\right) e^{-i\left(\nu^{\prime \prime \prime}-\nu^{\prime}\right) \phi_{E}} .
\end{aligned}
$$

In this equation, $d$ and $\phi_{E}$ refer to the radial and angular relative displacement between the centers of layers $i$ and $i-1$, and $k_{\rho}$ is the wavenumber of layer $i$. Once $\overline{\bar{N}}_{\nu \nu^{\prime}, i}^{\prime}$ is computed from (24), $\overline{\bar{N}}_{\nu \nu^{\prime},(i-1)}$ can be determined using a relation equivalent to (31) of Appendix A, i.e.

$$
\begin{aligned}
\overline{\bar{N}}_{\nu \nu^{\prime},(i-1)}= & \overline{\bar{\Gamma}}_{\nu(i-1)}^{+}+ \\
& \overline{\bar{T}}_{\nu(i-1)}^{-} \overline{\bar{N}}_{\nu \nu^{\prime}, i}^{\prime}\left[\overline{\bar{I}}-\overline{\bar{\Gamma}}_{\nu(i-1)}^{-} \overline{\bar{N}}_{\nu \nu^{\prime}, i}^{\prime}\right]^{-1} \overline{\bar{T}}_{\nu(i-1)}^{+} .
\end{aligned}
$$

The same procedure can then repeated from layer $i-1$ to layer $i-2$ and so on. The procedure ends at the borehole layer $i=0$ where the receiver antennas are located, to obtain $\overline{\bar{N}}_{\nu \nu^{\prime}, 0}$.

Note that there is no need here to effect the analogous procedure for obtaining $\bar{M}_{\nu \nu^{\prime}, i}$ (which in this case would proceed outwardly from the innermost layer to the outer layers) because in practice we are only interested in computing the fields in the borehole layer, and there is only one layer internal to it (the metallic mandrel, with $i=-1$ ), see Fig. 1.

The final expression for the fields in the borehole layer that generalizes (12) in the presence of modal coupling caused by the eccentric layers is of the form

$$
\left[\begin{array}{l}
e_{z \nu} \\
h_{z \nu}
\end{array}\right]= \begin{cases}{\left[\overline{\bar{H}}_{z \nu}\left(k_{\rho} \rho, \alpha\right) \overline{\bar{\Gamma}}_{\nu,-1}^{-}+\overline{\bar{J}}_{z \nu}\left(k_{\rho} \rho, \alpha\right)\right] \bar{b}_{\nu 0}} & \\
\overline{\bar{H}}_{z \nu}\left(k_{\rho} \rho, \alpha\right) \bar{a}_{\nu 0}+\overline{\bar{J}}_{z \nu}\left(k_{\rho} \rho, \alpha\right) \sum_{\nu^{\prime}=-\infty}^{+\infty} \overline{\bar{N}}_{\nu \nu^{\prime}, 0} \bar{a}_{\nu^{\prime} 0} & \rho>\rho_{T}\end{cases}
$$

where $\overline{\bar{\Gamma}}_{\nu,-1}^{-}$is the reflection matrix at the mandrel given by

$$
\overline{\bar{\Gamma}}_{\nu,-1}^{-}=\left[\begin{array}{cc}
-\frac{J_{\nu}\left(\alpha k \rho R_{-1}\right)}{H_{\nu}\left(\alpha k \rho R_{-1}\right)} & 0 \\
0 & -\frac{J_{\nu}^{\prime}\left(k \rho R_{-1}\right)}{H_{\nu}^{\prime}\left(k \rho R_{-1}\right)}
\end{array}\right] .
$$


TABLE I

TILTED MAGNETIC DIPOLES IN ECCENTRIC BOREHOLE

\begin{tabular}{|c|c|c|c|c|}
\hline Voltage $\left(\times 10^{-11}\right)$ & $\Re e\left[V_{r 1}\right]$ & $\Im m\left[V_{r 1}\right]$ & $\Re e\left[V_{r 2}\right]$ & $\Im m\left[V_{r 2}\right]$ \\
\hline Analytical result* & 13.916 & 524.71 & 9.7342 & 183.20 \\
\hline FEM result & 13.903 & 524.91 & 9.7116 & 183.26 \\
(rel. error) & $(-0.09 \%)$ & $(0.04 \%)$ & $(-0.23 \%)$ & $(0.03 \%)$ \\
\hline Proposed method & 13.900 & 524.73 & 9.7101 & 183.22 \\
(rel. error) & $(-0.11 \%)$ & $(0.00 \%)$ & $(-0.25 \%)$ & $(0.01 \%)$ \\
\hline
\end{tabular}

*benchmark

\section{Numerical Results}

Unless indicated otherwise, the logging tool geometry considered here consists of three tilted-coil antennas with 11.43 $\mathrm{cm}$ radius each, wrapped around a metallic mandrel with 10.16 $\mathrm{cm}$ in radius. One coil antenna functions as transmitter and the other two as receivers. The midpoints of the two receiver coils are located at $60.96 \mathrm{~cm}$ and $76.2 \mathrm{~cm}$ away from the midpoint transmiter coil along the $z$-direction shown in Fig. 1. The phase difference (PD) and amplitude ratio (AR) between the voltages measured at two receivers coils are the parameters of interest to estimate the formation conductivities. We assume the relative permeability and permittivity of all media to be unity.

\section{A. Magnetic Dipole Model}

We first validate the proposed method against a magnetic dipole model as considered in [17], [22]. In this case the mandrel is absent. In our model, we set the radius of coil antennas to $0.5 \mathrm{~cm}$ so that the fields produced by the tilted coils are well approximated by magnetic dipoles. The radius of the borehole is $10.16 \mathrm{~cm}$. All the coils are eccentric, with a displacement of $7.62 \mathrm{~cm}$ from the center of borehole along the same direction. The transmitter and receiver coils are tilted by $-45^{\circ}$ and $45^{\circ}$, respectively. The two receiver coils are positioned $30.46 \mathrm{~cm}$ and $43.18 \mathrm{~cm}$ away from transmitter coil, respectively. The borehole is filled by an isotropic fluid (mud filtrate) with $100 \Omega \cdot \mathrm{m}$ resistivity. The surrounding Earth formation consists of an anisotropic medium with horizontal and vertical resistivities equal to $0.1 \Omega \cdot \mathrm{m}$ and $4 \Omega \cdot \mathrm{m}$, respectively. The transmitter coil is excited by a $12 \mathrm{kHz}$ alternating current with $1 \mathrm{~A}$. Table I shows the voltages at the two receivers computed by three different approaches: an "analytical" method, as described in [17], [22], a numerical result based on the finite element method (FEM), and the proposed pseudoanalytical method. $\Re e$ and $\Im m$ denote the real and imaginary part of the voltages, respectively. The relative errors are below $0.25 \%$. Assuming the analytical method as benchmark, the pseudoanalytical results are in a slight better agreement.

\section{B. Tilted-Coil Antennas with Anisotropic Formation}

In this subsection, the effect of the anisotropy on the response of directional tools is investigated. The radius of the mandrel, coil antennas, and borehole are $10.16 \mathrm{~cm}, 11.43 \mathrm{~cm}$, and $12.7 \mathrm{~cm}$ respectively, all concentric. The vertical distances of the receiver coils to the transmitter coil are $60.96 \mathrm{~cm}$ and

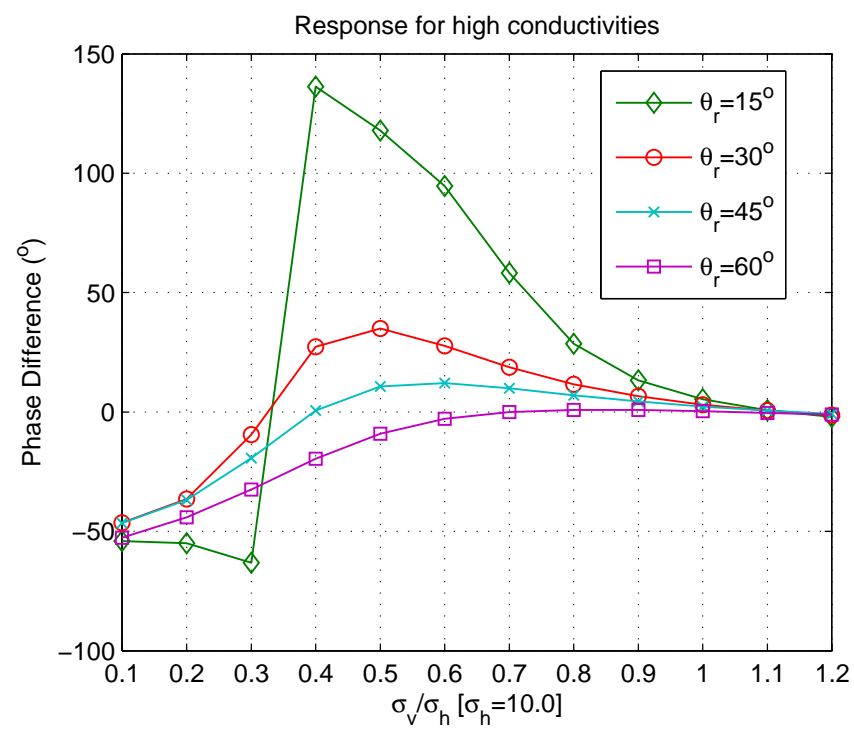

Fig. 3. Directional tool response (phase difference between receiver voltages) in an anisotropic formation versus anisotropy ratio: Higher conductivity case.

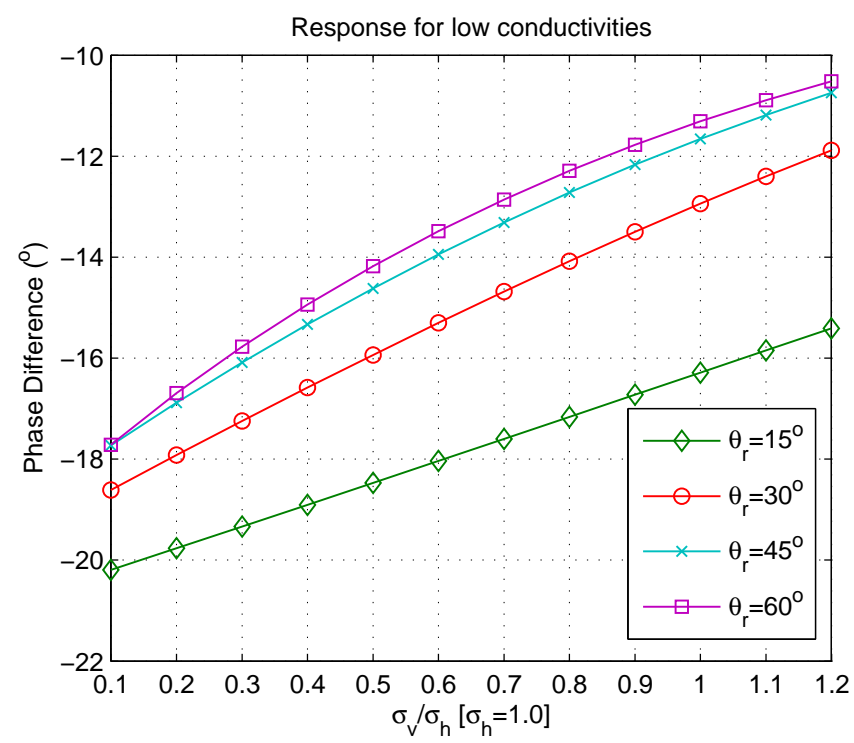

Fig. 4. Directional tool response (phase difference between receiver voltages) in an anisotropic formation versus anisotropy ratio: Lower conductivity case.

$76.2 \mathrm{~cm}$. The borehole is filled with an oil-based mud having $5 \times 10^{-4} \mathrm{~S} / \mathrm{m}$. The tilt angle of the $2 \mathrm{MHz}$ transmitter coil is fixed at $\theta_{T}=-45^{\circ}$, while four different tilt angles are considered for the two receivers: $\theta_{R}=15^{\circ}, 30^{\circ}, 45^{\circ}, 60^{\circ}$. Fig. 3 shows the PD as a function of the ratio of vertical and horizontal components of the formation conductivity (the horizontal conductivity is fixed at $\sigma_{h}=10.0 \mathrm{~S} / \mathrm{m}$, and the vertical conductivity is varied). In practice, the vertical conductivity is typically lower than the horizontal one. It can be seen that when $\theta_{R}=15^{\circ}$, the tool has maximum sensitivity to the anisotropy. Fig. 4 shows a similar result now for a formation with lower conductivity, $\sigma_{h}=1.0 \mathrm{~S} / \mathrm{m}$. Note the different scales used in Fig. 3 and Fig. 4, indicating that the tool is more sensitive to the anisotropy ratio for higher 


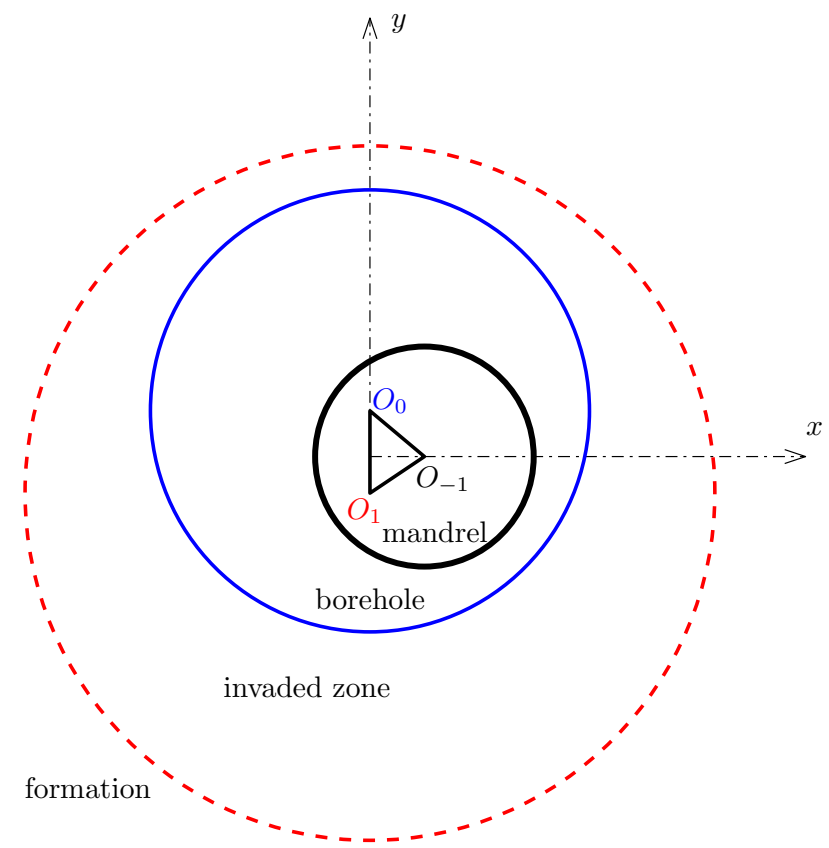

Fig. 5. Multieccentric environment with eccentric tool and eccentric invaded zone. $O_{-1}, O_{0}$, and $O_{1}$ refer to the centers of tool mandrel, borehole, and invaded zone respectively.

conductivities.

\section{Eccentric Tool in a Formation with Eccentric Invaded Zone}

We next consider a multieccentric problem with crosssection as depicted in Fig. 5. In this case, the mandrel is eccentric to the borehole, and the first formation layer is eccentric to both mandrel and borehole. This first layer may constitute an invaded zone caused by diffusion of the borehole fluid into formation, and having mixed electrical properties. The points $O_{-1}, O_{0}$, and $O_{1}$ indicate the centers of mandrel, borehole, and invaded zones, respectively. The corresponding radii are $R_{-1}=10.16 \mathrm{~cm}, R_{0}=22.86 \mathrm{~cm}$, and $R_{1}=38.1$ $\mathrm{cm}$. The radii of coil antennas are equal to $11.43 \mathrm{~cm}$. The tilted angles of transmitter and receiver coils are $-45^{\circ}$ and $45^{\circ}$ respectively, all of them tilted along the $x$-direction. The operation frequency is $2 \mathrm{MHz}$. The borehole is filled with oil-based mud having $\sigma=5 \times 10^{-4} \mathrm{~S} / \mathrm{m}$. The conductivity of formation is $10.0 \mathrm{~S} / \mathrm{m}$. We fix the mandrel eccentricity to $5.08 \mathrm{~cm}$ in the $y$-direction in Fig. 5, i.e. $\left|O_{0} O_{-1}\right|=2$ in and $\angle O_{1} O_{0} O_{-1}=0^{\circ}$. The magnitude of the eccentric offset of invaded zone $\left|O_{0} O_{1}\right|$ varies, but is also in the $y$ direction. Fig. 6 plots the results of PD and AR for different conductivities in the invaded zone, $\sigma \in\left[5 \times 10^{-4}, 10^{1}\right] \mathrm{S} / \mathrm{m}$, and for different offsets $\left|O_{0} O_{1}\right| \in[0,10] \mathrm{cm}$. It can be seen from Fig. 6 that the phase difference is less sensitive to the eccentric offset with an invaded zone having conductivity very close to either the borehole mud or earth formation conductivities. Fig. 6 (top) shows that the PD sensitivity to offset peaks at about $\sigma \approx 10^{0} \mathrm{~S} / \mathrm{m}$. In addition, Fig. 6 (bottom) shows that the AR varies more strongly with the invaded zone conductivity as the eccentric offset is increased. Fig. 7 is an inversion plot showing (PD,AR) traces as the eccentric offset
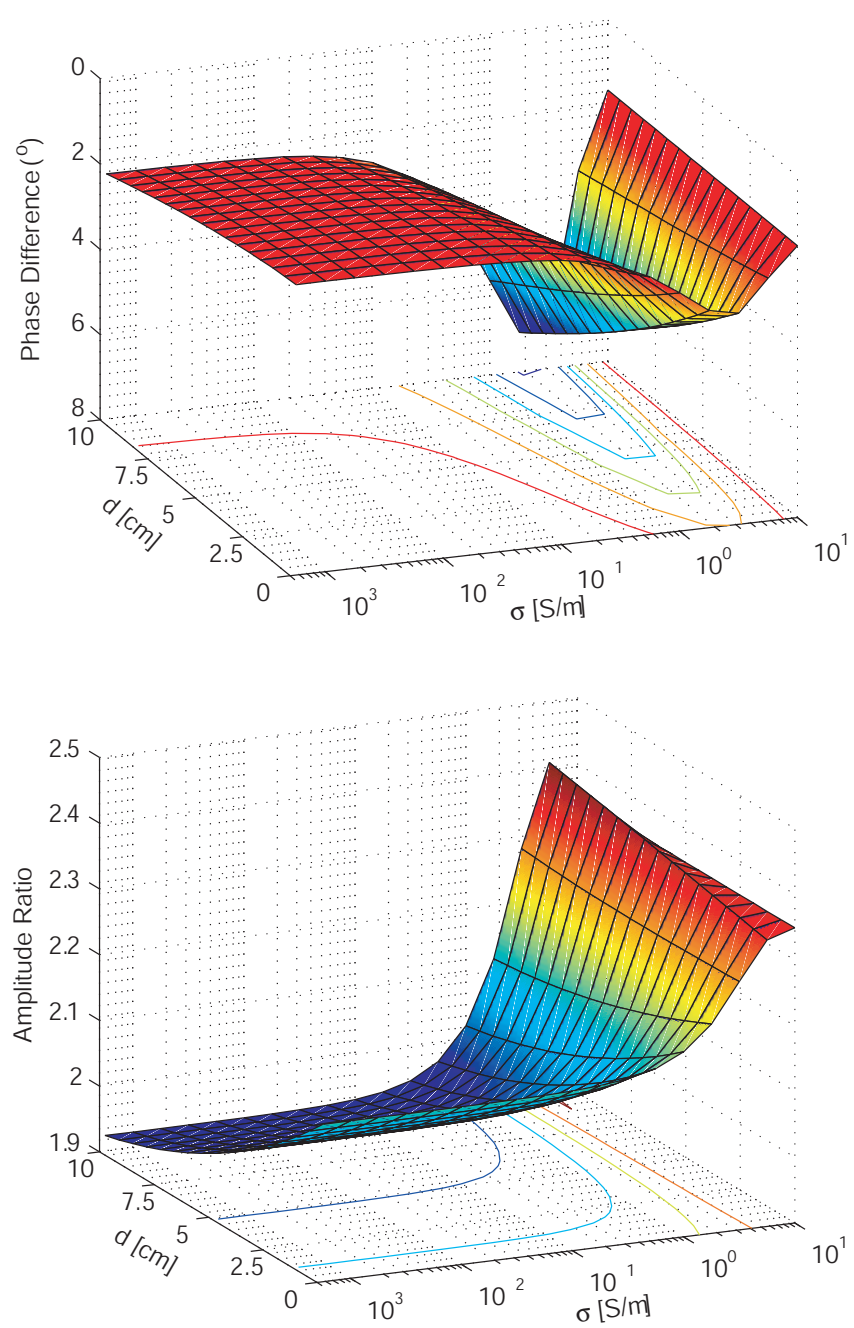

Fig. 6. Logging tool response (phase difference and amplitude ration between the two receiver voltages) in a multieccentric environment with invaded zone as depicted in Fig. 5. The eccentric offset of the tool with respect to the borehole is fixed and equal to $\left|O_{0} O_{-1}\right|=5.08 \mathrm{~cm}$. The parameter $d$ represent the eccentric offset of the invaded zone with respect to the borehole, $d=\left|O_{0} O_{-1}\right|$, which is varied between 0 and $10 \mathrm{~cm}$. The parameter $\sigma$ represents the conductivity of the invaded zone, which is varied between $\sigma=5 \times 10^{-4} \mathrm{~S} / \mathrm{m}$ (borehole conductivity) and $\sigma=10 \mathrm{~S} / \mathrm{m}$ (formation conductivity).

and invaded zone conductivity are varied. Note that the traces converge at the point $\sigma=10 \mathrm{~S} / \mathrm{m}$, as the conductivity of the invasion becomes equal to that of the external Earth formation.

\section{Directional Logging Example}

Directional LWD tools can provide azimuthal information about the surrounding formation by rotating the tilt azimuth angle $\phi_{T}$ along the drilling axis. As $\phi_{T}$ is varied to cover a $360^{\circ}$ angular span, the measured well-data can be used to determine the next drill direction for real-time geosteering purposes. Directional tools provide capability of detecting nearby beds given sufficient resistivity contrast [21]. In this subsection, we evaluate the azimuthal sensitivity of a directional LWD tool response when approaching a conductive bed boundary. 


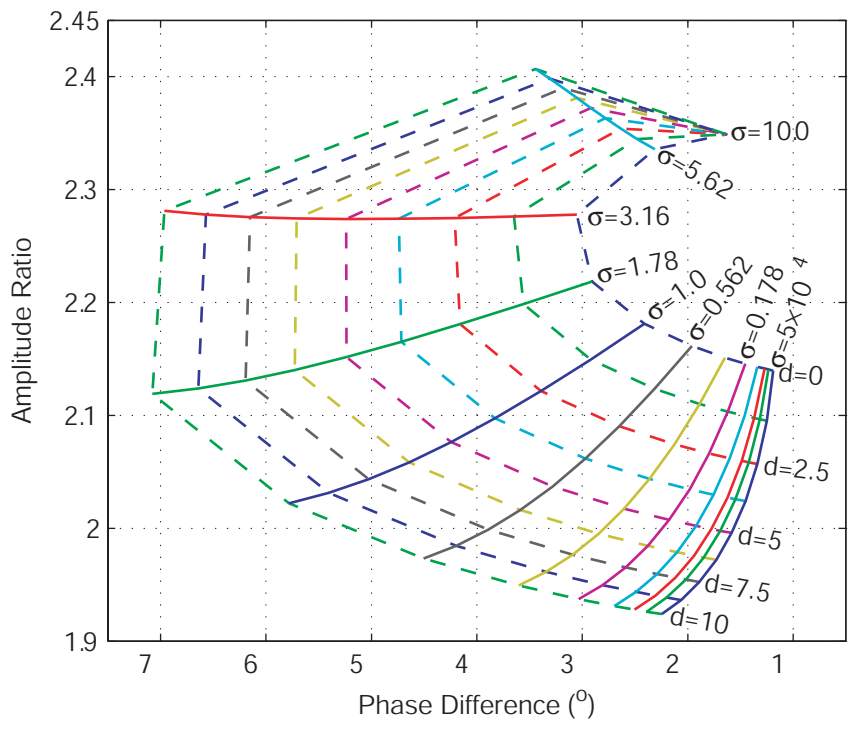

Fig. 7. Plot of eccentric offset $d$ between invaded zone and borehole in $\mathrm{cm}$ and invaded zone conductivity $\sigma$ in $\mathrm{S} / \mathrm{m}$ as function of the phase difference and amplitude ratio between the receiver voltages.

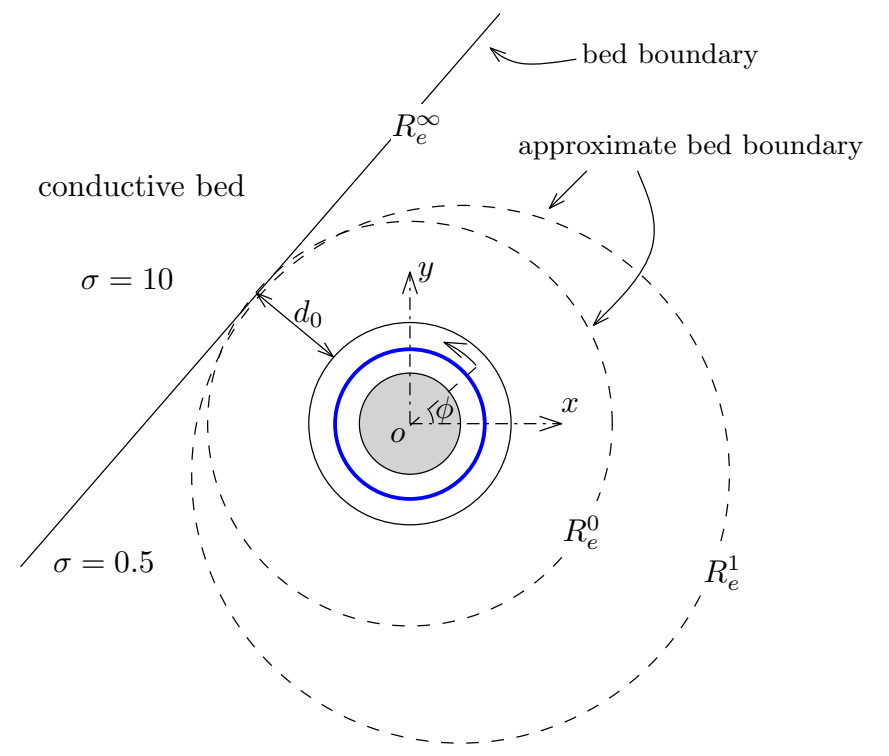

Fig. 8. The solid circles represent the mandrel (gray area), coil antennas (in blue color), and borehole boundary respectively, with radii of $10.16 \mathrm{~cm}, 11.43$ $\mathrm{cm}$, and $12.7 \mathrm{~cm}$, respectively. The mandrel and antennas rotate anticlockwise while drilling. A conductive bed with flat boundary is located at a distance of $68.6 \mathrm{~cm}$ to the borehole boundary along the $135^{\circ}$ direction. The dashed eccentric circles are used to approximate the bed boundary (for sufficiently large radius).

We use an example similar to the one considered in [21]. We assume a conductive bed located in the proximity of the logging tool along the $135^{\circ}$ direction, as shown in Fig. 8. The bed is assumed to have an approximately flat boundary, which can be approximated by a circle of sufficiently large radius as illustrated in Fig. 8. While drilling occurs, the driller can be made to rotate $360^{\circ}$ on its axis, thus providing variation on $\phi_{T}$. We assume the elevation tilt angles $\theta_{T}$ of the transmitter and receivers to be fixed, as specified below. We consider a one-transmitter, two-receiver LWD tool, with the receiver coils

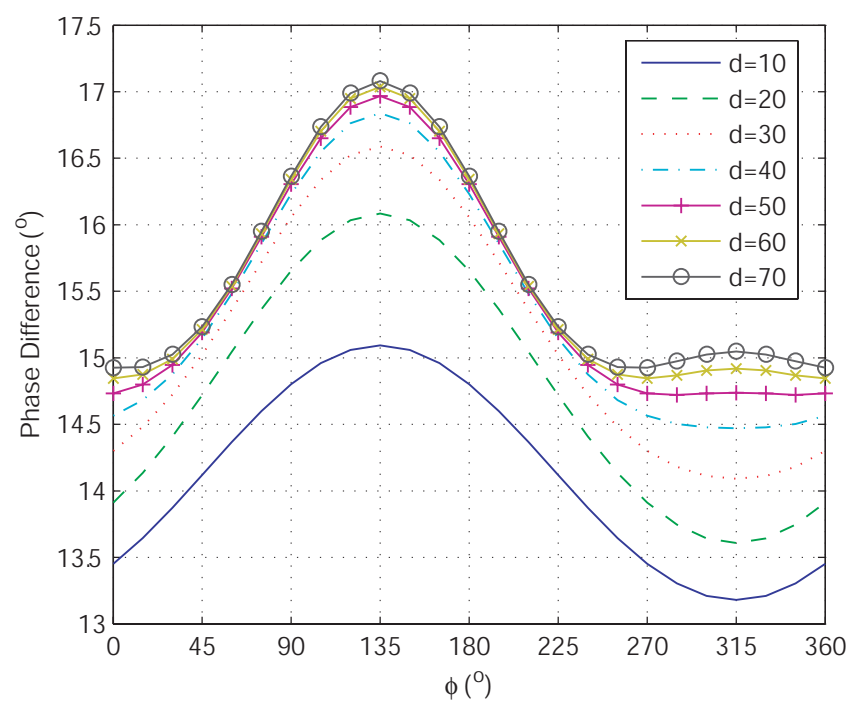

Fig. 9. Azimuthal response (phase difference) of directional LWD tool, where $d$ denotes the eccentric offset in $\mathrm{cm}$. The tool rotates throughout a period of $360^{\circ}$. The response converges for large $d$, and peaks at $\phi_{T}=135^{\circ}$ which is angle corresponding to (the nearest point of) the bed boundary.

arranged $60.96 \mathrm{~cm}$ and $76.2 \mathrm{~cm}$ away from the transmitter coil. The bed boundary is located $68.6 \mathrm{~cm}$ away from the borehole boundary, i.e. $d_{0}=68.6 \mathrm{~cm}$ in Fig. 8 . We use a series of eccentric circles $R_{e}^{d}$ (with $d$ denoting the eccentric offset in $\mathrm{cm})$ to approximate the bed boundary. The other parameters of this problem are as follows. The conductivities of borehole, intervening formation, and conductive bed are $5 \times 10^{-4} \mathrm{~S} / \mathrm{m}$, $0.5 \mathrm{~S} / \mathrm{m}$, and $10 \mathrm{~S} / \mathrm{m}$, respectively. The radii of mandrel, coil antennas, and borehole are $10.16 \mathrm{~cm}, 11.43 \mathrm{~cm}$, and $12.7 \mathrm{~cm}$, respectively, and the tool is concentric to the borehole. The elevation tilt angles $\theta_{T}$ of transmitter, bottom receiver, and top receiver are $-45^{\circ}, 0^{\circ}$, and $45^{\circ}$, respectively, and the operating frequency is $2 \mathrm{MHz}$. Fig. 9 shows the PD response for different $\phi_{T}$ and $R_{e}^{d}$. The results clearly converge as the eccentric offset $d$ of the approximating circle is increased. The PD response reaches an extrema at $\phi_{T}=135^{\circ}$, precisely when the azimuth tilt angle points in the direction of (the nearest point of) the bed boundary.

\section{CONCLUSIONS}

A pseudoanalytical formulation to analyze resistivity logging tools developed in [25] was extended to anisotropic and multieccentric cylindrically-layered Earth formations, where sucessive cylindrical layers may have different axes of symmetry. A recursive approach based on a successive application of Graf's addition theorem from the outermost layer to the borehole layer is developed to account for multiple eccentric layers external to the borehole and to yield generalized reflection matrices associated to a fictitious boundary concentric to the logging tool. This allows for an efficient iterative approach to be used for computing the fields due to a tilted-coil source within the borehole layer. Various numerical examples are shown to validate the method and to illustrate its applicability for hydrocarbon exploration, in particular in the modeling of 
formation resistivity log responses and in the detection of lateral beds.

\section{APPENDIX A: GENERALIZED REFLECTION AND TRANSMISSION MATRICES}

Here, we extend the methodology in [22] to compute local reflection and transmission coefficients between anisotropic cylindrical layers. Assuming that a standing wave is incident from medium $i+1$ onto the boundary $R_{i}$, continuity of transverse field components gives

$$
\begin{aligned}
& {\left[\begin{array}{l}
\overline{\bar{J}}_{z \nu}\left(k_{\rho_{i+1}} R_{i}, \alpha_{i+1}\right) \\
\overline{\bar{J}}_{\phi \nu}\left(k_{\rho_{i+1}} R_{i}, \alpha_{i+1}\right)
\end{array}\right]=} \\
& {\left[\begin{array}{ll}
\overline{\bar{J}}_{z \nu}\left(k_{\rho_{i}} R_{i}, \alpha_{i}\right) & -\overline{\bar{H}}_{z \nu}\left(k_{\rho_{i+1}} R_{i}, \alpha_{i+1}\right) \\
\bar{J}_{\phi \nu}\left(k_{\rho_{i}} R_{i}, \alpha_{i}\right) & -\overline{\bar{H}}_{\phi \nu}\left(k_{\rho_{i+1}} R_{i}, \alpha_{i+1}\right)
\end{array}\right]\left[\begin{array}{c}
\overline{\bar{T}}_{\nu i}^{-} \\
\overline{\bar{\Gamma}}_{\nu i}^{-}
\end{array}\right],}
\end{aligned}
$$

see Fig. 10(a). Similarly, assuming that an outgoing wave is incident from medium $i$ onto the boundary $R_{i}$, the continuity of transverse field components gives

$$
\begin{aligned}
& {\left[\begin{array}{l}
\overline{\bar{H}}_{z \nu}\left(k_{\rho_{i}} R_{i}, \alpha_{i}\right) \\
\overline{\bar{H}}_{\phi \nu}\left(k_{\rho_{i}} R_{i}, \alpha_{i}\right)
\end{array}\right]=} \\
& \quad\left[\begin{array}{ll}
\overline{\bar{H}}_{z \nu}\left(k_{\rho_{i+1}} R_{i}, \alpha_{i+1}\right) & -\overline{\bar{J}}_{z \nu}\left(k_{\rho_{i}} R_{i}, \alpha_{i}\right) \\
\overline{\bar{H}}_{\phi \nu}\left(k_{\rho_{i+1}} R_{i}, \alpha_{i+1}\right) & -\overline{\bar{J}}_{\phi \nu}\left(k_{\rho_{i}} R_{i}, \alpha_{i}\right)
\end{array}\right]\left[\begin{array}{c}
\overline{\bar{T}}_{\nu i}^{+} \\
\overline{\bar{\Gamma}}_{\nu i}^{+}
\end{array}\right],
\end{aligned}
$$

see Fig. 10(b). In (28) and (29), $\overline{\bar{\Gamma}}_{\nu i}^{+}$and $\overline{\bar{\Gamma}}_{\nu i}^{-}$are the $2 \times 2$ local reflection matrices at the boundary $R_{i}$, while $\overline{\bar{T}}_{\nu i}^{+}$and $\overline{\bar{T}}_{\nu i}^{-}$are the $2 \times 2$ local transmission matrices at $R_{i}$.

Following [5], [14], recursive relations to obtain the matrices $\bar{M}_{\nu i}$ and $\bar{N}_{\nu i}$ in (12) are given by

$$
\begin{gathered}
\overline{\bar{M}}_{\nu(i+1)}=\overline{\bar{\Gamma}}_{\nu i}^{-}+\overline{\bar{T}}_{\nu i}^{+} \overline{\bar{M}}_{\nu i}\left[\overline{\bar{I}}-\overline{\bar{\Gamma}}_{\nu i}^{+} \overline{\bar{M}}_{\nu i}\right]^{-1} \overline{\bar{T}}_{\nu i}^{-}, \\
\overline{\bar{N}}_{\nu i}=\overline{\bar{\Gamma}}_{\nu i}^{+}+\overline{\bar{T}}_{\nu i}^{-} \overline{\bar{N}}_{\nu(i+1)}\left[\overline{\bar{I}}-\overline{\bar{\Gamma}}_{\nu i}^{-} \overline{\bar{N}}_{\nu(i+1)}\right]^{-1} \overline{\bar{T}}_{\nu i}^{+} .
\end{gathered}
$$

Note that the matrices $\overline{\bar{M}}_{\nu i}$ and $\overline{\bar{N}}_{\nu i}$ are computed from the innermost to the outermost layer and vice-versa, respectively.

In conductive layers, the coefficient matrices of (28) and (29) include exponentially large as well as exponentially small terms. This can cause numerical instabilities for large arguments. It is beneficial to normalize the solutions as

$$
\left[\begin{array}{l}
e_{z \nu} \\
h_{z \nu}
\end{array}\right]=\overline{\bar{H}}_{z \nu}^{n}\left(k_{\rho} \rho, \alpha\right) \bar{a}_{\nu}^{n}\left(k_{\rho} \rho, \alpha\right)+\overline{\bar{J}}_{z \nu}^{n}\left(k_{\rho} \rho, \alpha\right) \bar{b}_{\nu}^{n}\left(k_{\rho} \rho, \alpha\right),
$$

with $\bar{a}_{\nu}^{n}\left(k_{\rho} \rho, \alpha\right)=\overline{\bar{H}}_{z \nu}\left(k_{\rho} \rho, \alpha\right) \bar{a}_{\nu}, \quad \bar{b}_{\nu}^{n}\left(k_{\rho} \rho, \alpha\right)=$ $\overline{\bar{J}}_{z \nu}\left(k_{\rho} \rho, \alpha\right) \bar{b}_{\nu}$, and $\overline{\bar{H}}_{z \nu}^{n}\left(k_{\rho} \rho, \alpha\right)=\overline{\bar{J}}_{z \nu}^{n}\left(k_{\rho} \rho, \alpha\right)=I_{2 \times 2}$. Similarly, we rewrite the azimuthal components as

$$
\left[\begin{array}{l}
e_{\phi \nu} \\
h_{\phi \nu}
\end{array}\right]=\overline{\bar{H}}_{\phi \nu}^{n}\left(k_{\rho} \rho, \alpha\right) \bar{a}_{\nu}^{n}\left(k_{\rho} \rho, \alpha\right)+\overline{\bar{J}}_{\phi \nu}^{n}\left(k_{\rho} \rho, \alpha\right) \bar{b}_{\nu}^{n}\left(k_{\rho} \rho, \alpha\right),
$$

where

$$
\begin{aligned}
\overline{\bar{H}}_{\phi \nu}^{n}\left(k_{\rho} \rho, \alpha\right) & =\overline{\bar{H}}_{\phi \nu}\left(k_{\rho} \rho, \alpha\right) \overline{\bar{H}}_{z \nu}^{-1}\left(k_{\rho} \rho, \alpha\right), \\
\overline{\bar{J}}_{\phi \nu}^{n}\left(k_{\rho} \rho, \alpha\right) & =\overline{\bar{J}}_{\phi \nu}\left(k_{\rho} \rho, \alpha\right) \overline{\bar{J}}_{z \nu}^{-1}\left(k_{\rho} \rho, \alpha\right) .
\end{aligned}
$$

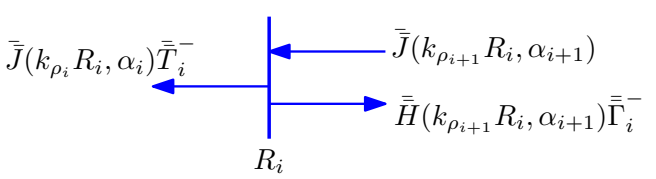

(a)

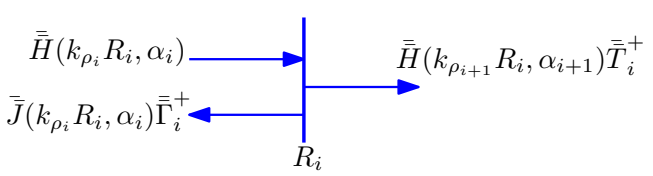

(b)

Fig. 10. Boundary conditions in terms of reflection and transmission coefficients for (a) standing and (b) outgoing waves, respectively. (a) A standing wave is incident from layer $i+1$ onto boundary $R_{i}$, an outgoing wave is reflected and a standing wave is transmitted into layer $i$. (b) An outgoing wave is incident from layer $i$ onto boundary $R_{i}$, a standing wave is reflected and an outgoing wave is transmitted into layer $i+1$.

The normalized matrices are better conditioned than the original ones. The fields in the borehole are expressed as

$$
\left[\begin{array}{c}
e_{z \nu} \\
h_{z \nu}
\end{array}\right]=\left\{\begin{array}{c}
{\left[\overline{\bar{H}}_{z \nu}^{n}\left(k_{\rho} \rho, \alpha\right) \overline{\bar{M}}_{\nu 0}^{n}+\overline{\bar{J}}_{z \nu}^{n}\left(k_{\rho} \rho, \alpha\right)\right] \bar{b}_{\nu 0}^{n}\left(k_{\rho} \rho, \alpha\right)} \\
\rho \leq \rho_{T} \\
{\left[\overline{\bar{H}}_{z \nu}^{n}\left(k_{\rho} \rho, \alpha\right)+\overline{\bar{J}}_{z \nu}^{n}\left(k_{\rho} \rho, \alpha\right) \overline{\bar{N}}_{\nu 0}^{n}\right] \bar{a}_{\nu 0}^{n}\left(k_{\rho} \rho, \alpha\right)} \\
\rho>\rho_{T}
\end{array}\right.
$$

where

$$
\begin{aligned}
& \overline{\bar{N}}_{\nu i}=\overline{\bar{J}}_{z \nu}^{-1}\left(k_{\rho} \rho, \alpha\right) \overline{\bar{N}}_{\nu i}^{n} \overline{\bar{H}}_{z \nu}\left(k_{\rho} \rho, \alpha\right) \\
& \overline{\bar{M}}_{\nu i}=\overline{\bar{H}}_{z \nu}^{-1}\left(k_{\rho} \rho, \alpha\right) \overline{\bar{M}}_{\nu i}^{n} \overline{\bar{J}}_{z \nu}\left(k_{\rho} \rho, \alpha\right)
\end{aligned}
$$

\section{APPENDIX B: ITERATIVE SOLUTION}

Generalized reflection matrices in multieccentric media can be obtained through a recursive application of Graf's addition theorem. After the innermost fictitious boundary is introduced, the final geometry becomes equivalent to that of a problem consisting of mandrel, coil antennas, and one fictitious outer boundary (all concentric). Similar to [5], we obtain in the borehole layer,

$$
\bar{b}_{\nu 0}-\sum_{\nu^{\prime}=-\infty}^{+\infty} \overline{\bar{N}}_{\nu \nu^{\prime}, 0} \overline{\bar{M}}_{\nu^{\prime}, 0} \bar{b}_{\nu^{\prime} 0}=\bar{C}_{\nu}^{-}+\sum_{\nu^{\prime}=-\infty}^{+\infty} \overline{\bar{N}}_{\nu \nu^{\prime}, 0} \bar{C}_{\nu^{\prime}}^{+},
$$

where $\overline{\bar{M}}_{\nu, 0}=\overline{\bar{\Gamma}}_{\nu,-1}^{-}$is the reflection matrix of the steel mandrel [4], [5], and $\bar{C}_{\nu}^{ \pm}$are $2 \times 1$ column vectors given by [5]

$$
\begin{aligned}
\bar{C}_{\nu}^{ \pm} & =\frac{-i \pi}{2} c_{T E}\left[\begin{array}{c}
\frac{i \omega \mu \nu}{k_{z}} G_{\nu}\left(k_{\rho} \rho_{T}\right) \\
k_{\rho} \rho_{T} G_{\nu}^{\prime}\left(k_{\rho} \rho_{T}\right)
\end{array}\right] \\
c_{T E} & =i^{\nu} J_{\nu}\left(k_{z} \rho_{T} \tan \theta_{T}\right) e^{-i\left(k_{z} z_{T}+\nu \phi_{T}\right)}
\end{aligned}
$$

where $G_{\nu}$ stands for $J_{\nu}$ with (+) superscript or $H_{\nu}$ with (-) superscript.

Direct solve for $\bar{b}_{\nu 0}$ in (37) involves a matrix inversion, which can become ill-conditioned for large $k_{z}, d$, and/or $\alpha$. We can instead write an iterative scheme

$$
\bar{b}_{\nu 0}^{l+1}=\bar{C}_{\nu}^{-}+\sum_{\nu^{\prime}=-\infty}^{+\infty} \overline{\bar{N}}_{\nu \nu^{\prime}, 0}\left(\bar{C}_{\nu^{\prime}}^{+}+\overline{\bar{M}}_{\nu^{\prime}, 0} \bar{b}_{\nu^{\prime} 0}^{l}\right) .
$$


for $l=0,1, \ldots$. The first term $\bar{C}_{\nu}^{-}$in the right-hand side corresponds the initial wave excited by the transmitter coil (homogeneous solution). The second term $\overline{\bar{N}}_{\nu \nu^{\prime}, 0} \bar{C}_{\nu^{\prime}}^{+}$is the (incoming) reflected wave produced by (reflection of the initial outgoing wave from) the external boundary. Iteration (39) starts with $\bar{b}_{\nu 0}^{0}=0$; then $\bar{b}_{\nu 0}^{1}$ is solution of the wave traveling across borehole once (without mandrel effect); $\bar{b}_{\nu 0}^{2}$ is solution the wave traveling across borehole twice (reflected once by the mandrel); $\bar{b}_{\nu 0}^{l}$ is the solution of the wave traveling in the borehole $l$ times. An adaptive stop criterion can be set up for this iterative process. The above fixed-point iterative procedure is convergent because the elements of $\overline{\bar{N}}_{\nu \nu^{\prime}, 0}$ and $\bar{M}_{\nu^{\prime}, 0}$ correspond to reflection coefficients with magnitude less than one. A detalied discussion on convergence criteria of iterative procedures such as above is presented [28] from a more general standpoint.

\section{APPENDIX C: FURTHER NUMERICAL CONSIDERATIONS}

The integral in (10) can be accelerated by subtracting the homogeneous solution [5]. A possible way to evaluate the relative error introduced by truncating $k_{z}$ is to estimate the ratio $\varepsilon\left(k_{z}\right)=\left|e_{z \nu}\left(k_{z}\right)-e_{z \nu}^{h}\left(k_{z}\right)\right| /\left|e_{z \nu}^{h}\left(k_{z}\right)\right|$, where $e_{z \nu}^{h}$ refers to the homogeneous solution. Combining the second term for $\rho \leq \rho_{T}$ in (26) with the first term in (39), we have $\left[e_{z \nu}^{h}, h_{z \nu}^{h}\right]^{T}$ $=\overline{\bar{J}}_{z \nu}\left(k_{\rho} \rho, \alpha\right) \bar{C}_{\nu}^{-}$. For the nonhomogeneous contribution to be sufficiently small, the last two terms in (39) and the first term in (26) (take $\rho=\rho_{T}$ ) should approach zero. For typical tool geometries, the outgoing wave term in (26) dominates those from (39) for large $\left|k_{z}\right|$. Hence, the error can be estimated by the ratio of outgoing and incoming wave contributions in (26). Assuming $\alpha=1$ and using (27), we have $\varepsilon\left(k_{z}\right) \simeq$ $\left(H_{\nu}\left(k_{\rho} \rho_{T}\right) J_{\nu}\left(k_{\rho} R_{-1}\right)\right) /\left(J_{\nu}\left(k_{\rho} \rho_{T}\right) H_{\nu}\left(k_{\rho} R_{-1}\right)\right)$, which leads to the asymptotic estimate $k_{z}^{\mathrm{m}} \geq \ln \varepsilon\left(k_{z}\right) /\left(2 R_{-1}-2 \rho_{T}\right)$ for large $k_{z}$. Here, $k_{z}^{\mathrm{m}}$ denotes the truncating point for $k_{z}$ in the numerical integration and we assume a PEC mandrel [5]. The above estimate agrees with prior numerical investigations [25].

For truncating Graf's series, we note that the argument of the Bessel functions is $k_{\rho} d$, whose largest value occurs for large $k_{z}$, when $\left|k_{\rho} d\right| \simeq\left|k_{z} d\right|$. Since Bessel functions decrease rapidly for increasing order and fixed argument when the absolute value of the order becomes larger than the absolute value of the argument, the series can be truncated at order $\nu^{\mathrm{m}} \simeq\left|k_{z}^{\mathrm{m}} d\right|$. Using the above estimate and some typical values for desired accuracy and geometrical parameters, say $R_{-1} \simeq 10 \mathrm{~cm}, \rho_{T} \simeq 12 \mathrm{~cm}, d \simeq 12 \mathrm{~cm}$, we have $\left|\nu^{\mathrm{m}}\right| \simeq 12$. This number of terms was found to be sufficient in most of the examples considered here.

\section{ACKNOWLEDGMENT}

We thank Dr. Burkay Donderici from Halliburton Energy Services for providing validation data.

\section{REFERENCES}

[1] M. Sato, J. Fuziwara, M. Miyairi, K. Kashihara, and H. Nitsuma, Directional induction logging methods Proceedings of the SPWLA 35th Annual Logging Symposium, pp. 1-16, Jun. 19-22, 1994.
[2] M. Bittar, Electromagnetic wave resistivity tool having a tilted antenna for determining the horizontal and vertical resistivities and relative dip angle in anisotropic earth formations, U.S. Patent 6,163,155, Dec. 19, 2000.

[3] T. Hagiwara and H. Song, Directional resistivity measurements for azimuthal proximity detection of bed boundaries, U.S. Patent 6,181,138, Jan. 30, 2001.

[4] T. Hagiwara, E. J. Banning, R. M. Ostermeier, and S. M. Haugland, "Effects of mandrel, borehole, and invasion for tilt-coil antennas SPE 78th Annual Technical Conference and Exhibition, paper SPE 84254, Denver, Colorado, Oct. 5-8, 2003.

[5] Y. K. Hue and F. L. Teixeira, "Analysis of tilted-coil eccentric borehole antennas in cylindrical multilayered formations for Well-Logging applications," IEEE Trans. Antennas Propag., vol. 54, no. 4, pp. 1058-1064, Apr. 2006.

[6] J. R. Wait, Geo-Electromagnetism, Academic Press, New York, 1982.

[7] T. Wang and S. Fang, "3-D electromagnetic anisotropy modeling using finite differences," Geophys., vol. 66, no. 5, pp. 1386-1398, 2001.

[8] Y. K. Hue, F. L. Teixeira, L. S. Martin, and M. S. Bittar, "Threedimensional simulation of eccentric LWD tool response in boreholes through dipping formations," IEEE Trans. Geosci. Remote Sens., vol. 43, no. 2, pp. 257-268, Feb. 2005.

[9] H. O. Lee and F. L. Teixeira, "Cylindrical FDTD analysis of LWD tools through anisotropic dipping-layered earth media," IEEE Trans. Geosci. Remote Sens., vol. 45, no. 2, pp. 383-388, Feb. 2007.

[10] D. Wu, J. Chen, and C. R. Liu, "An efficient FDTD method for axially symmetric LWD environments," IEEE Trans. Geosci. Remote Sens., vol. 46, no. 6, pp. 652-656, Jun. 2008.

[11] D. Pardo, L. Demkowicz, C. Torres-Verdn, and M. Paszynski, "Twodimensional high-accuracy simulation of logging-while-drilling (LWD) measurements using a self-adaptive goal-oriented hp finite element method," SIAM J. Appl. Math., vol. 66, no. 6, pp. 2085-2106, 2006.

[12] D. Pardo, L. Demkowicz, C. Torres-Verdn, C. and Michler, "PML enhanced with a self-adaptive goal-Oriented hp finite-element method: simulation of through-casing borehole resistivity measurements," SIAM J. Sci. Comput., vol. 30, no. 6, pp. 2948-2964, 2008.

[13] M. S. Novo, L. C. Silva, and F. L. Teixeira, "Finite volume modeling of borehole electromagnetic logging in 3-D anisotropic formations using coupled scalar-vector potentials," IEEE Antennas Wireless Propag. Lett., vol. 6, pp. 549-552, 2007.

[14] J. R. Lovell and W. C. Chew, "Response of a point source in a multicylindrically layered medium," IEEE Trans. Geosci. Remote Sens., vol. GE-25, no. 6, pp. 850-858, Nov. 1987.

[15] J. R. Lovell and W. C. Chew, "Effect of tool eccentricity on some electrical well-logging tools," IEEE Trans. Geosci. Remote Sens., vol. 28, no. 1, pp. 127-136, Jan. 1990.

[16] H. Wang, P. So, S. Yang, W. J. R. Hoefer, and H. Du, "Numerical modeling of multicomponent induction well-logging tools in the cylindrically stratified anisotropic media," IEEE Trans. Geosci. Remote Sens., vol. 46, no. 4, pp. 1134-1147, Apr. 2008.

[17] L. Zhong, J. Li, A. Bhardwaj, L. C. Shen, and R. C. Liu, "Computation of triaxial induction logging tools in layered anisotropic dipping formations," IEEE Trans. Geosci. Remote Sens., vol. 46, no. 4, pp. 1148-1163, Apr. 2008.

[18] S. Ebihara and Y. Inoue, "Analysis of eccentered dipole antenna for borehole radar," IEEE Trans. Geosci. Remote Sens., vol. 47, no. 4, pp. 1073-1088, Apr. 2009.

[19] G. L. Wang, C. Torres-Verdin, and S. Gianzero, "Fast simulation of triaxial borehole induction measurements acquired in axially symmetrical and transversely isotropic media," Geophysics, vol. 74, no. 6, pp. e233e249, Nov.-Dec. 2009.

[20] M. S. Bittar, "Electromagnetic wave resistivity tool having a tilted antenna for determining the horizontal and vertical resistivities and relative dip angle in anisotropic earth formations," U.S. Patent 7,557,579 B2, Jul. 7, 2009.

[21] L. Chou, Q. Li, A. Darquin, J.-M. Denichou, R. Griffiths, N. Hart, A. McInally, G. Templeton, D. Omeragic, I. Tribe, K. Watson, and M. Wiig, "Steering toward enhanced production," Oilfield Review, vol. 17, no. 3, pp. 54-63, Oct. 2005.

[22] W. C. Chew, Waves and Fields in Inhomogeneous Media, IEEE Press, Piscataway NJ, 1995.

[23] L. W. Pearson, "A construction of the fields radiated by z-directed point sources of current in the presence of a cylindrically layered obstacle," Radio Sci., vol. 21, no. 4, pp.559-569, Jul.-Aug. 1986.

[24] W. C. Chew, "The singularities of a Fourier-type integral in a multicylindrical layer problem," IEEE Trans. Antennas Propag., vol. AP-31, no. 4, pp.653-655, Jul. 1983. 
[25] Y. K. Hue, "Analysis of electromagnetic well-logging tools," Ph.D. dissertation, The Ohio State Univ., Columbus, OH, 2006.

[26] M. Abramowitz and I. A. Stegun, Handbook of Mathematical Functions, p. 363., National Bureau of Standards, Applied Mathematics Series - 55, Tenth printing, 1972.

[27] N. J. Vilenkin, Special Functions and the Theory of Group Representations, Amer. Math. Soc., Providence, RI, 1968.

[28] M. DUrso, I. Catapano, L. Crocco, and T. Isernia, "Effective solution of 3-D scattering problems via series expansions: Applicability and a new hybrid scheme," IEEE Trans. Geosci. Remote Sens., vol. 45, no. 3, pp. 639-648, Apr. 2007.

Guo-Sheng Liu was born in Hubei Province, China, in 1983. He received the B.S. degree of Science and Ph.D degree of Engineering, both from South China University of Technology (SCUT), Guangzhou, China, in 2005 and 2010, respectively. From 2008 to 2010, he was a visiting scholar with the ElectroScience Laboratory, the Ohio State University, Columbus. Since 2011, he has been as an Assistant Professor at the Department of Information Management Engineering, the Guangdong University of Technology, Guangzhou, China. His current research interests include finite-difference time-domain (FDTD) methods and numerical modeling of well-logging tools.

Fernando L. Teixeira received the Ph.D degree in Electrical Engineering from the University of Illinois at Urbana-Champaign in 1999. From 1999 to 2000 he was a Postdoctoral Associate with the Massachusetts Institute of Technology. In 2000 he joined the The Ohio State University, where he is now a Professor with the Department of Electrical and Computer Engineering and also affiliated with the ElectroScience Laboratory.

His current research interests include modeling of electromagnetic sensors for hydrocarbon exploration, time-domain computational electromagnetics, metamaterials, and ultrawideband inverse scattering. He received a number of awards for his research, including the CAREER Award from the National Science Foundation, the triennial Booker Fellowship from the International Union of Radio Science (USNC/URSI), and the Outstanding Young Engineer Award from the IEEE Microwave Society (MTT-S). Dr. Teixeira has served as chair of the Joint IEEE AP/MTT-S Columbus Chapter and currently serves as Associate Editor for the IEEE Antennas and Wireless Propagation Letters.

Guo-Ji Zhang received the B.S. degree in Computer Science and Applications and the Ph.D. degree in Circuit and Systems from South China University of Technology (SCUT), Guangzhou, China, in 1977 and 1999, respectively.

Since 1977 he has done research work at SCUT, where he is currently a Professor with the Department of Mathematics. His research interests include computational electromagnetics and computational intelligence. 